\title{
Unresectable Skin Carcinoma
}

National Cancer Institute

\section{Source}

National Cancer Institute. Unresectable Skin Carcinoma. NCI Thesaurus. Code C157324.

Skin carcinoma that is not amenable to surgical resection. 